nau analysiert ist. Dr. med. Theodor Byrckmann in Köln erhielt durch Vermittlung der Rosenkreuzer in Wien etwa fünfundzwanzig Jahre nach Paracelsi Tod einen Abzug des Hirschvogel-Porträts 1540 zusammen mit dem sogenannten «Kärntner Trio »(1538) der Defensiones, des Labyrinthus, der letzten Tartarusarbeit, und der Chronik des Landes Kärnten. Ohne die Bemühungen der Kölner Rosenkreuzer wäre dieses Trio, das unter den Standesakten von Klagenfurt vergraben und vergessen war, verloren gegangen. Vom Holzschneider Franz Hoogenbergh ließ Dr. med. Th. Byrckmann nach Erhalt des Trios mit Plattenabzug in der väterlichen Druckerei Byrckmannis Erben in beiden oberen Bildquadranten rosenkreuzerische Adeptenzeichnungen anbringen: links die Geburt zum irdischen Licht, signiert mit Kreuz und Rosa, rechts die symbolisch sehr interessant dargestellte Geburt des vorgeschrittenen Adepten zum himmlischen Licht (die zwei «Lichter» Hohenheims). Dieses unzweideutige Signet beweist, daß die Rosenkreuzer schon fast hundert Jahre vor dem vorgeschützten Christian Rosenkreuz existiert haben, deren Wirken durch die Druckoffizin Byrckmann in Köln über die von Lechler-Feyerabend in Frankfurt am Main, die alte «Pfaffenstraße» des Rheins über Straßburg und die Wasserstraße der Donau (Neuburg mit den Paracelsischen Manuskriptensammlungen) nach Wien und Schlesien (Montanus) reichte und u.a. das Kärntner Trio gerettet hat. Die Ergebnisse meiner früheren Arbeit über «PARAcelsus und die Rosenkreuzer» im III. Band der NAP sind heute allgemein anerkannt, so daß Francis Kordas, F.R.C., in mehreren Artikeln im «Rosicrucian Digest» 1950 über «PARAcelsus the Rosicrucian» schreiben konnte: Few people know that Paracelsus was not only one of the greatest mystics of the Middle Ages, but also an initiated Rosicrucian.»

\title{
Ein unbekannter Brief von Johann Heinrich Lambert an Johannes Gessner
}

Von Max Steck, zurzeit Basel

1. Vor genau hundert Jahren hat der durch seine astronomischen Forschungen wie durch seine Biographien zur Kulturgeschichte der Schweiz gleich bedeutende Forscher Rudolf WoLF in der berühmten Briefsamm- 
lung OtT-Usteri in Zürich einen wichtigen Brief Johann Heinrigh LamBerts (1728-1777) an Johannes Gessner (1709-1790) wiedergefunden und veröffentlicht ${ }^{1}$. Er konnte damit eine Lücke, welche in dem von Johann (III.) Bernouldi nach Lamberts Tode herausgegebenen Deutschen gelehrten Briefwechsel Lamberts noch verblieben war ${ }^{2}$, schließen und eine für die wissenschaftliche Biographie Lamberts und Gessners gleich bedeutsame neue Quelle aufweisen.

2. Bei der gegenwärtigen Durcharbeitung des gesamten handschriftlichen Nachlasses LAMBERTS auf der Universitätsbibliothek Basel mußte ich vielfach auch den handschriftlichen Nachlaß Daniel Hubers (1768-1829), Professor der Mathematik und Bibliothekar in Basel, des ersten bedeutenden Lambert-Biographen, heranziehen. Ich fand dabei u.a. auch in diesem Nachlaß eine von Daniel Huber nach dem ihm damals (1828) vorliegenden Original angefertigte genaue handschriftliche Abschrift eines weiteren, bisher gänzlich unbekannten und in seiner Existenz auch nicht vermuteten Briefes Lamberts an Johannes Gessner ${ }^{3}$, der ein ebenso bedeutsames Quellendokument für die LAMBERT- und Gessner-Forschung darstellt wie der Fund von Rudolf Wolf vor hundert Jahren. Auch dieser, seiner Existenz nach bisher völlig unbekannte Brief, stammt aus der Briefsammlung Oтt-Usteri in Zürich und wurde, wie aus dem folgenden Notiztext Daniel Hubers (St. Nr. 47, Bl. 1 recto) hervorgeht, im Jahre 1828 von Herrn Staatsrat UsterI in Zürich im Original an D.HuBER geschickt mit der Bitte um Weiterleitung als Geschenk an die Bibliothek der Geburtsstadt LAMBERTS, dem damals (1728) der Eidgenossenschaft zugewandten Mülhausen, wo man 1828 eine Jubelfeier zur hundertsten Wiederkehr des Geburtstages LAMBERTS veranstaltete. Die genannte Notiz hat folgenden Wortlaut: «Copie zweier Briefe von Lambert an Joh. Gessner, nach den Originalen ausgefertigt, welche Hr. Staatsrath Usteri mir übersandt hatte, um sie nach Müllhausen zu schicken. 1828.»4

${ }^{1}$ R. Wolf, Ein verloren geglaubter Brief Lamberts an Johannes Geßner, Mitteilungen der naturforschenden Gesellschaft in Bern, 1851, p. 37-40.

${ }^{2}$ Band II, Berlin 1782, p.174ff. - Der Brief ist dort nach Nr.XIV. (p.176/7) einzuschalten.

${ }^{3}$ Daniel Huber, Handschriftlicher Nachlaß auf der Universitätsbibliothek in Basel, sign. Mscr. L. Ib. 83 b. Stück Nr. 4.

${ }^{4}$ Der andere Brief ist eine genaue Abschrift des schon von JoH. (III.) Bernoulli als Nr. XIII in seiner Edition der Briefe Lamberts (s. Fußnote 2) abgedruckten französischen Briefes vom 28. November 1758, Chur. (Der Ort fehlt im Druck.) 
Auf Anfrage aus Anlaß der Erstellung meiner Bibliographia Lambertiana $^{5}$ hat mir die «Leitung des Historischen Museums von Mülhausen» unter dem 18. Dezember 1941 mitgeteilt, daß in Mülhausen weder im Stadtarchiv, noch in der Bibliothek, noch im Historischen Museum ungedruckte Lambertiana vorhanden sind. Demnach muß das Original des hier mitzuteilenden Briefes LAMBERTS als verloren gelten.

3. Die nach dem Originalbrief genommene wörtliche Abschrift des mitzuteilenden Briefes lautet bei Daniel Huber unter Zeilenangabe der HuBERschen Handschrift wörtlich so:

(St. $47, \mathrm{Bl} .2$ recto):

à Mr. Gesner Dr. en M. Prof. en Phys. membre de L'Acad. de Berlin, d'Upsal. etc. Zuric.

HochEdelgebohrner, Hochgelehrtester Herr! Insonders HochgeEhrter Herr!

Euer HochEdelgeboren habe Ursache dehmütigst abzubitten, daß ich bis dahin verschoben, für die mir zu Zürch erwiesene schätzbare Gewogenheit, und mitgegebenen Brief an Herrn Prof. J.Bernoully den gebührenden Dank zu erstatten, $u$. bedaure, daß ich mich noch nicht in solchen

10 Umständen befinde, da [ss] meine geringen Dienste ein Pfand meiner Erkenntlichkeit sey[n] könnten.

Einige kleine Hausgeschäfte werden mich hier noch etwas Zeit aufhalten, die sich noch mehr verlängern könnte, wenn ich in der Nähe Gelegenheit finde, meine Photometrie drucken zu lassen, weil es mir ziemlich gleichgültig ist, wo ich mich bis zum Frieden aufhalte.

Seit dem Euer HochEdelgeboren die Güte gehabt mir die Scheuchzerischen Observationen zukommen zu lassen, habe ich in dem Commercio literario noch andre gefunden $u$.

20 aufgeschrieben, die H Professor Doppelmayer zu Nürnberg von 1732 bis 1742 angestellt, $u$. welche desto genauer sind, da kein Tag dabei fehlt, $u$. die Höhen des Barometers bis auf ${ }^{1 / 12}$ einer linie angegeben sind. Ich habe daraus die Tage genommen, an welchen der Mond in Apogaeo u. Perigaeo ist, nebst dem Tag so vorhergeht und den drei folgenden, um die mittle-

5 Enthalten in M.Steck, Johann Heinrich Lambert. Schriften zur Perspektive, Berlin 1943, p. 93-154; auch separat, Berlin 1943. 
re Höhe des Quecksilbers [im Original Lamberss Zeichen für Hg] zu finden. Aus 730 Observationen, finde keinen

27 so großen Unterschied, daß dem Mond eine $1 / 4$ linie könnte

(Bl.2, verso)

zugeschrieben werden, $u$. wenn man von diesen 11 Jahren ein einziges $\mathrm{Jahr}$ wegläßt, so kann man nach Belieben die mittlere

30 Höhe des Quecksilbers [w. oben, Zeile 26] im Apogaeo oder im Perigaeo größer und kleiner

finden. Da diese die Hofnung ganz wegnimmt, einen Theil der barometrischen Veränderungen auf Periodo zu bringen, die von dem Laufe des des Mondes [Mondzeichen im Original] abhiengen, so bleibt nichts

mehr, als die Ursache davon in der Sonnenwärme u. auf Erden zu suchen, und es wird ungemein schwer fallen, allgemeine Gesetze davon zu bestimmen. Das einige ist, daß man die Schranken und das Mittel finde, wie es in der Astronomie geschehen. Nach diesen kann man etwan auch von den Anomalien Gründe und Regeln suchen.

40 Der Herr Prof. Huber zu Basel, dem ich einen Besuch abstattete [*] hat den Cometen den 2ten May und 14. Tage nachher gesehen. Er hatte davon aus dem HalleJI Tractate einen Riß gemacht, welcher mit dem meinigen vollkommen übereinkommt, u. den Cometen observierte er damals zwischen dem Becher und dem Sextans Urania. Er war sehr klein und hatte keinen merklichen Schweif, weil derselbe von der Erde ziemlich weggekehrt war. Den 2 ten May strich er an dem Horizont vorbey, und nachgehends war er höher, weil er sich in dem Sextanten

50 dem $\ddot{A}$ quator näherte. Dermalen wird man ihn wegen

51 der Entfernung höchstens nur durch Ferngläser sehen

(Bl. 3 recto)

können, zwischen dem Regulo und corda Hydra, etwas näher bei dem letztern. Man hat zu Basel und hier den Abendstern dafür gehalten, u. weil er nahe bei dem Horizont war, durch einen bloß optischen Betrug der Augen geglaubt, daß er sich sichtbar bewege.

[*] Es handelt sich um den Vater Daniel H., Johann Jacob Huber, den Astronomen, welcher 1798 verstorben ist. 
Von der Wirkung des Cometen glaubt man, das

Jahr werde um etliche Secunden länger, u. die obliqui-

taet der Eccliptic gleichfalls um etwas verändert

60 seyn. Seine Wirkung auf das Wasser muß un-

merklich gewesen seyn, weil der Comet so gar klein

ist.

Ich habe die Ehre mit nochmaliger Dankbezeugung

und wahrer Ehrerbietung zu seyn

Euer HochEdelgeboren

unterthänigst $\mathrm{u}$. gehorsamster

Müllhausen den Diener

68

6. Jun. 1759.

JH LAMBERT.

\section{Buchbesprechungen}

1. Henry E.Sigerist, A History of Medicine. Volume I: Primitive and Archaic Medicine. (Publication No 27 Historical Library Yale Medical Library.) Oxford University Press, London, Geoffrey Cumberledge, 1951. Sh. 45-/. 564, Seiten, 48 Tafeln mit 104 Abbildungen.

Mit diesem ersten der auf 7-8 Bände berechneten Medizingeschichte, die eine erstmalig in diesem Umfang unternommene Weltgeschichte der Medizin darstellt, beginnt ein Standardwerk zu erscheinen, daß mit Sartons Monumentalwerk, seiner History of Science, in Parallele tritt und doch so ganz andere Ziele in Darstellung und Sinngebung verfolgt, daß sie als zwei völlig verschiedengeartete Werke in Erscheinung treten - ebenso verschiedenartig wie nach Temperament und Charakter die beiden Verfasser, die sich, soweit dies heute schon gesagt werden kann, in ihren Werken in glücklicher Weise ergänzen werden.

Sigerist stellt sich von allem Anfang an konsequent auf den Boden der praktischen Medizin und untersucht historisch ihre soziale Funktion: Medizin in ihrer gesundheitsfördernden, vorbeugenden, heilenden, wiederherstellenden Funktion. Dies wohlverstanden im breiten Rahmen der historischen, religiösen und philosophischen Bedingtheiten der verschiedenen Epochen, d. h. unter Berücksichtigung jener geistigen Kräfte und Mächte, denen von Anbeginn an, und namentlich im Beginn der Menschheitsentwicklung, die «Theorie» der Medizin weitgehend unterworfen war. Was Sigerist in seiner Darstellung in den Vordergrund rückt, ist das soziale Gefüge der Gesellschaft, ist die soziale Stellung des Kranken in den verschiedenen Zeiten, angefangen mit seiner Stellung in vorgeschichtlicher Zeit und bei den heutigen Primitiven, im alten Ägypten und Mesopotamien. Damit ist gleichzeitig der Inhalt des ersten Bandes kurz umrissen. Ein Überblick über die geographisch-wirtschaftlichen und sozialen Verhältnisse der betreffenden 